\section{Variaciones de tendencia, ciclos y volatilidad del precio de la madera en pie de eucalipto y pino en Entre Ríos para el período 2001-2020}

Variations in the trend, cycles and volatility of the price of standing timber of eucalyptus and pine in Entre Ríos for the period 2001-2020

\section{Néstor Albino Molina | Luis María Mestres}

\section{RESUMEN}

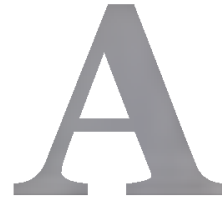

rgentina cuenta con 1.3 millones de hectáreas de bosque implantadas, siendo las provincias de Misiones, Corrientes y Entre Ríos las de mayor superficie, en total el 78,2\% de la superficie nacional. A partir de datos relevados por el INTA EEA Concordia, el objetivo es analizar el comportamiento estacional de precios de monte en pie de eucalipto y pino con corteza en el período 2001-2020. La serie de precios forestal para monte en pie de eucalipto y pino en pesos históricos fue calculada en dólares de acuerdo al tipo de cambio de ese día y luego se ajustó por el Índice de Precios al Consumidor-Estados Unidos, obteniéndose así una serie nominal y otra real. Al realizar el análisis de tendencia que muestra la serie de largo plazo, se observa un movimiento creciente con pendiente o,081 en eucalipto y 0,029 en pino, que se mantiene en toda la serie. El período estudiado se compone de 5 ciclos con una duración entre 3 y 5 años. Los ciclos no poseen periodicidades iguales. El componente estacional muestra las fluctuaciones de un precio forestal dentro de un año calendario. Finalizando, el componente estacionalidad, formado por variaciones sin patrón regular o identificable. La tendencia no muestra un patrón regular de comportamiento, ya que los precios suben, se mantienen o bajan imprevistamente.

Palabras clave: pino; eucalipto; precios, serie de tiempo.

\section{ABSTRACT}

Argentina has 1.3 million hectares of implanted forest, being the provinces of Misiones, Corrientes and Entre Ríos the ones with the
Néstor Albino Molina molina.nestor@inta.gob.ar

INTA EEA Bella Vista, Corrientes Luis María Mestres mestres.luis@inta.gob.ar

INTA EEA Bella Vista, Corrientes ARGENTINA

\section{COMO CITAR ESTE ARTÍCULO}

Molina N. A. , Mestres L. M. (2021). Variaciones de tendencia, ciclos y volatilidad del precio de la madera en pie de eucalipto y pino en Entre Ríos para el período 2001-2020. Revista de la Facultad de Ciencias Económicas, $27(2), 35-47$.

http://dx.doi.org/10.30972/rfce.2725657 
largest surface, in total $78.2 \%$ of the national surface. Based on data collected by INTA EEA Concordia, the objective is to analyze the seasonal behavior of prices of eucalyptus and pine with bark standing forest in the period 2001-2020. The series of forest prices for standing eucalyptus and pine in historical pesos was calculated in dollars according to the exchange rate of that day and then adjusted by the Consumer Price Index-United States, thus obtaining a nominal series and another real. When performing the trend analysis shown by the longterm series, an increasing movement with a slope of 0.081 in eucalyptus and 0.029 in pine is observed, which is maintained throughout the series. The period studied is made up of 5 cycles with a duration between 3 and 5 years. The cycles do not have equal periodicities. The seasonal component shows the fluctuations of a forest price within a calendar year. Ending, the seasonality component, made up of variations with no regular or identifiable pattern. The trend does not show a regular pattern of behavior, as prices rise, stay or fall unexpectedly.'

Keywords: pine; eucalyptus; prices; time series.

\section{INTRODUCCIÓN}

Argentina cuenta con 1,3 millones de hectáreas de bosque implantadas (Peirano et. al., 2019), compuestos casi exclusivamente por pinos (Pinus sp.), eucaliptos (Eucalyptus sp.), sauces (Salix sp.) y álamos (Populus sp.), de los cuales el 78,2\% se concentra en la Mesopotamia (provincias de Misiones, Corrientes y Entre Ríos) (MAGyP, 2015). De las 860 mil ha de las plantaciones de pino, un $82.2 \%$ se encuentra en esta región, así como un $87.8 \%$ de las 314 mil ha de eucalipto, (MAGyP, 2015), Tabla 1. En general, se estiman 42 mil puestos de trabajo directos y 68.700 indirectos generados por la cadena forestal (Peirano et al 2019).

Tabla 1. Superficie forestal en Argentina

\begin{tabular}{|c|c|c|c|c|}
\hline & Pino & Eucalipto & Resto & Total \\
\hline Misiones & 348305 & 40902 & 16617 & 405824 \\
\hline Corrientes & 345965 & 121857 & 6161 & 473983 \\
\hline Entre Rios & 14156 & 112785 & 23856 & 150797 \\
\hline Resto & 152924 & 38325 & 95940 & 287189 \\
\hline Total País & 861350 & 313869 & 142574 & 1317793 \\
\hline
\end{tabular}

Fuente: Peirano et al (2019).

El objetivo del presente trabajo es analizar el comportamiento estacional de precios de plantaciones en pie de eucalipto y pino con corteza para el período 2001-2020, a partir de datos relevados por el INTA EEA Concordia (Vera, 2020 y Vera et al. 2020). 
La serie de precios presentada es representativa del Nordeste de Entre Ríos: Concordia, Federación y Colón.

Los efectos estacionales observados en precios, $y$ en menor medida en cantidades, corresponden a consideraciones de oferta y demanda. Estas fluctuaciones fueron estudiadas por Brescia y Rivera (2000) para frutas y hortalizas para un período de 15 años. Otro trabajo similar, y más reciente es un estudio para la producción frutícola regional, en un período de igual longitud, realizado por investigadores económicos del INTA Corrientes y Tucumán, (Molina y Ríos, 2019).

Fundamentos teóricos:

- Una serie de tiempo es una secuencia ordenada de observaciones realizada a través del tiempo en intervalos iguales, periódicos y consecutivos; aunque se pueden utilizar otras dimensiones, como el espacio (Wei, 2006).

- Los hechos que generan estos datos forestales incorporan la influencia de comportamiento de la economía, de factores climáticos y otros fenómenos aleatorios. Su principal componente es la estacionalidad, es decir, la forma como la serie de precios se comporta de manera sistemática en un período con intervalos de datos mensuales.

- Los modelos que describen series temporales son procesos estocásticos, es decir, procesos controlados por leyes probabilísticas. La construcción de un modelo de serie de tiempo depende de varios factores, tales como el comportamiento del fenómeno o el conocimiento a priori de su naturaleza y el objetivo de análisis. En la práctica, dependen también de métodos apropiados de estimación y de software adecuado (Morettin y Toloi, 2006).

- Modelo Clásico Multiplicativo. Para determinar la naturaleza de las fluctuaciones del precio, se calcularon los componentes de tendencia, estacional, cíclico y aleatorio. Las fluctuaciones de los precios fueron estudiadas por diversos autores (Gujarati y Porter, 2010 y Hoffmann 2006) que usaron un método de análisis cuantitativo que permitió desagregar los componentes de la serie. El modelo clásico de series temporales supone que una serie temporal de precios Pt, es el producto de cuatro componentes: tendencia, ciclo, estacionalidad y volatilidad.

\section{2 MATERIALES Y MÉTODOS}

\section{La Teoría Económica de la Serie de Tiempo}

Índice de Precios. En este trabajo se analiza el comportamiento de los precios medios mensuales de monte en pie de eucalipto y pino con corteza mediante el cálculo de índices de estacionalidad. Se calcularon precios promedios mensuales obtenidos de los registros del INTA EEA Concordia (Vera, et al, 2020). 
La base de datos utilizada son precios mensuales durante 20 años, período 2001-20201. Los precios históricos se convirtieron a su cotización del dólar estadounidense, ajustados por el índice de precios al consumidor estadounidense, obteniendo una serie de tiempo de valores reales.

Para la estacionalidad de la serie de precios se adoptó el modelo clásico de estacionalidad en series temporales.

El modelo multiplicativo (Hanke y Wichern, 2010) está dado por los siguientes factores.

El factor $T$ es la tendencia a largo plazo de una serie de tiempo; es la persistencia a largo plazo del patrón de aumento o disminución de precios, ocasionado por cambios en la tecnología, población e ingresos, y su duración es de muchos años.

Es una recta mínima cuadrática que refleja la evolución de la serie de tiempo que no sea influenciada por el ciclo económico. Una vez representada la serie, se ajusta a la línea más conveniente calculándose la pendiente de la recta, es el método más usado.

El factor $C$ representa las variaciones cíclicas alrededor de la tendencia, y se define como ondas de prosperidad, recesión, depresión y crecimiento; sufre la influencia de las interacciones de los factores que inciden en la actividad económica agraria, su duración va de dos a cinco años, con diferente intensidad.

El factor $E$ o variaciones estacionales, son fluctuaciones interanuales (ocurren cada 12 meses) ocasionados por condiciones climáticas, costumbres sociales, etc.

$Y$ el factor $V$ es el componente de volatilidad, midiendo las variaciones irregulares de una serie de tiempo, después de eliminar los otros componentes. Son eventos imprevisibles como sequía, inundación, helada, aparición de plagas y enfermedades, etc., y posee una corta duración y no se repite con frecuencia.

Son sistemáticos los factores tendencia, ciclo y estacional y no sistemático la volatilidad. Lo anterior se puede expresar como:

$$
P t=T t * C t * E t * V t
$$

Dónde:

- P: serie temporal de precios;

- T: componente de tendencia;

- C: componente cíclico;

- E. componente estacional;

- V: componente aleatorio;

- $\quad \mathrm{t}$ : los subíndices corresponde al año y mes.

${ }^{1}$ Los mismos pueden ser solicitados al INTA Concordia, Sector de economía: Cr. Luis Vera (vera.luis@inta.gob.ar) 
La serie de precios forestal para monte en pie de eucalipto y pino en pesos históricos fue calculada en dólares de acuerdo al tipo de cambio de ese día ${ }^{2}$ y luego se ajustó por el Îndice de Precios al Consumidor de Estados Unidos ${ }^{3}$, obteniéndose así una serie nominal y otra real.

Precio Histórico y Ajustado. Se realiza una presentación descriptiva de los datos, según medidas centrales y de dispersión. El precio promedio durante el periodo 2001-2020 para una tonelada de eucalipto en pie fue de U\$S19.55 y pino USS 18.97, el subperíodo de precio mayor para ambos bienes fue 2011-2015, el subperíodo de precio menor para ambos bienes fue 2001-2005.

Las tablas 2 y 3 muestran las estadísticas centrales y de dispersión de los precios en dólares de eucalipto y pino, para el período 2001-2020 y subperíodos en lustros.

Tabla 2. Estadísticas de la serie Precio Eucalipto, en dólares ajustado al IPC EEUU

\begin{tabular}{|c|c|c|c|c|c|c|c|}
\hline Período & Media & Desv. Est. & Mediana & Mínima & Período & Máxima & Período \\
\hline $2001-2020$ & 19.55 & 9.45 & 16.68 & 5.68 & $\mathrm{Ago} / 2002$ & 38.77 & Dic/2015 \\
\hline $2001-2005$ & 9.39 & 2.40 & 9.01 & 5.68 & $\mathrm{Ago} / 2002$ & 15.47 & $\mathrm{Dic} / 2001$ \\
\hline $2006-2010$ & 12.77 & 2.36 & 16.33 & 11.57 & $\mathrm{Feb} / 2006$ & 20.70 & $\mathrm{Dic} / 2010$ \\
\hline $2011-2015$ & 30.04 & 5.40 & 29.64 & 18.95 & $\mathrm{May} / 2011$ & 38.77 & $\mathrm{Dic} / 2015$ \\
\hline $2016-2020$ & 22.43 & 9.23 & 21.82 & 10.27 & $\mathrm{Dic} / 2020$ & 35.65 & $\mathrm{Jun} / 2016$ \\
\hline
\end{tabular}

Fuente: Elaboración propia en base a Vera, et al, 2020 y Banco Mundial (2020).

Tabla 3. Estadísticas de la serie Precio Pino, en dólares ajustado al IPC EEUU

\begin{tabular}{|c|c|c|c|c|c|c|c|}
\hline Período & Media & Desv. Est. & Mediana & Mínima & Período & Máxima & Período \\
\hline $2001-2020$ & 18.97 & 7.02 & 20.26 & 7.40 & Oct/2002 & 33.62 & Oct/2013 \\
\hline $2001-2005$ & 11.92 & 3.29 & 10.89 & 7.40 & Oct/2002 & 20.55 & Dic/2001 \\
\hline $2006-2010$ & 15.92 & 2.52 & 20.51 & 14.37 & Feb/2006 & 24.46 & Jul/2008 \\
\hline $2011-2015$ & 27.43 & 3.18 & 26.94 & 20.21 & May/2011 & 33.62 & Oct/2013 \\
\hline $2016-2020$ & 16.53 & 6.46 & 15.72 & 8.60 & Dic/2020 & 26.77 & Jun/2016 \\
\hline
\end{tabular}

Fuente: Elaboración propia en base a Vera, et al, 2020 y Banco Mundial (2020).

En las Figuras 1a) y 1b) se observa la evolución de los precios de eucalipto y pino en dólares históricos y ajustados por el IPC de Estados Unidos.

\footnotetext{
${ }^{2}$ Se utilizó el conversor de divisas OANDA https://www1.oanda.com/lang/es/currency/converter/

${ }^{3}$ Banco Mundial (2020) https://datos. bancomundial.org/indicador/FP.CPI.TOTL.ZG ?locations=US
} 
$40<$

Néstor Albino Molina | Luis María Mestres

Variaciones de tendencia, ciclos y volatilidad del precio de la madera en pie de eucalipto y pino...

Págs. 35 - 47. DOI: http://dx.doi.org/10.30972/rfce.2725657

Figura 1a. Evolución de Precios Monte Pie de Eucalipto. Histórico y Ajustado

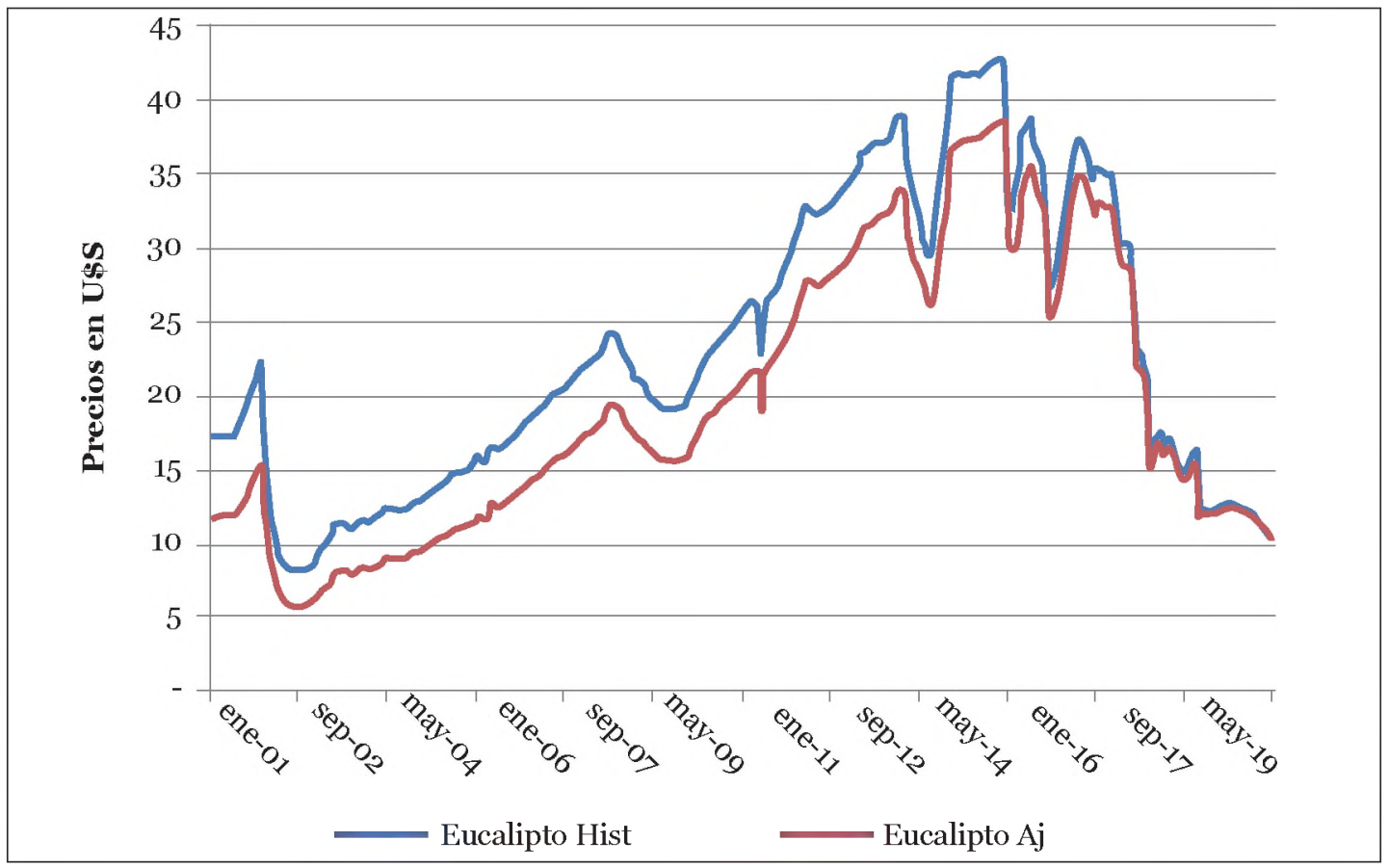

Fuente: Elaboración propia en base a Vera et al (2020).

Figura 1b. Evolución de Precios Monte Pie de Pino. Histórico y Ajustado

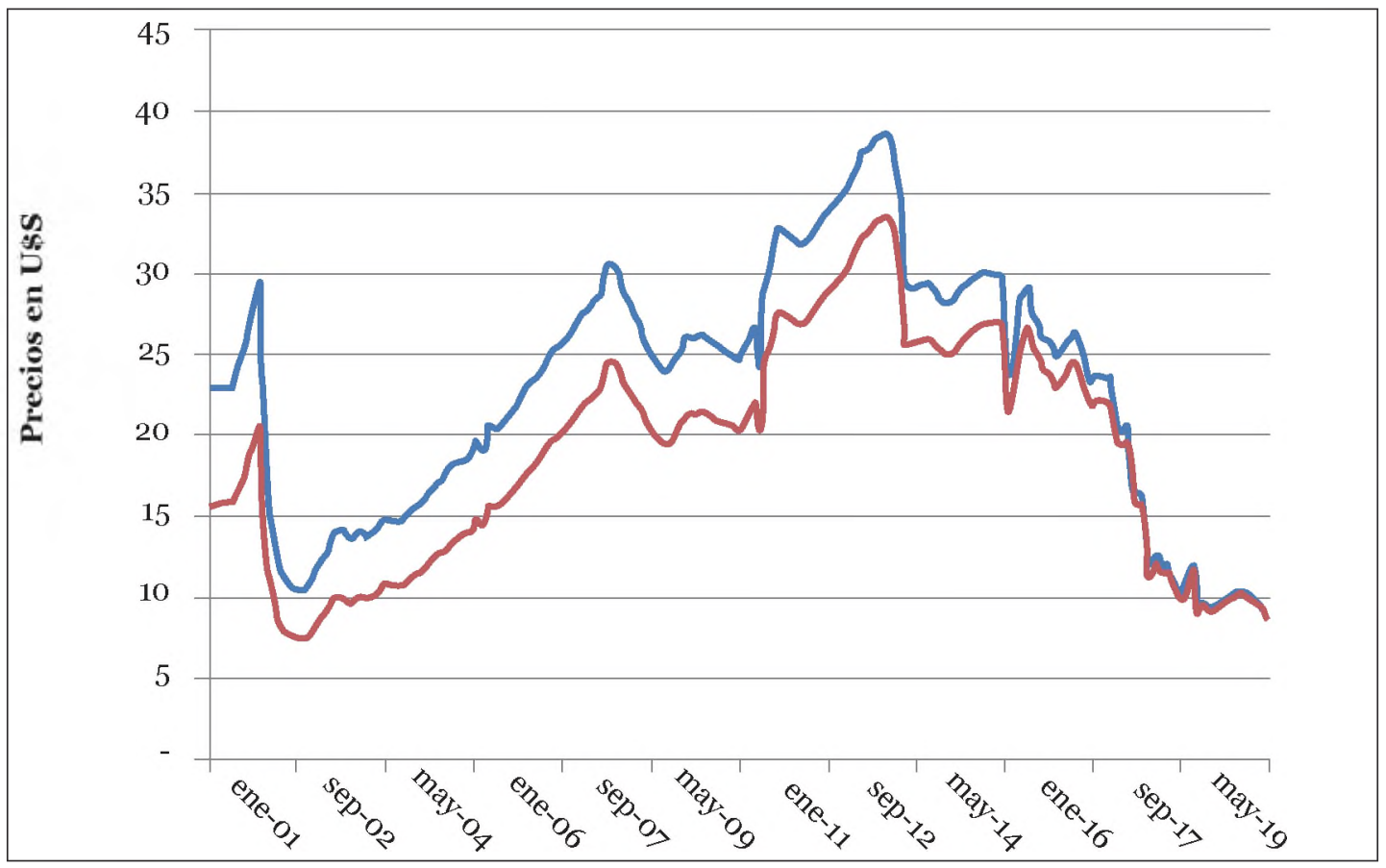

Fuente: Elaboración propia en base a Vera et al (2020).

REVISTA DE LA FACULTAD DE CIENCIAS ECONÓMICAS - UNNE - Argentina, Volumen 27 Núm. 2, julio-diciembre 2021, ISSN 1668-6365

http://revistas.unne. edu.ar/index.php/rfce 
La serie nos indica momentos en que los precios del monte en pie son inferiores a su precio de largo plazo (subvaluación del commodity) y superiores al de largo plazo (sobrevaluación).

En particular, la figura 2 muestra que el período subperíodos de subvaluación entre enero de 2001 y julio 2010, repitiéndose en junio 2018 a diciembre 2020. Hay un subperíodo de sobrevaluación entre agosto de 2010 y mayo 2018.

Figura 2. Períodos de Sobrevaluación y Subvaluación de los precios Eucalipto y Pino

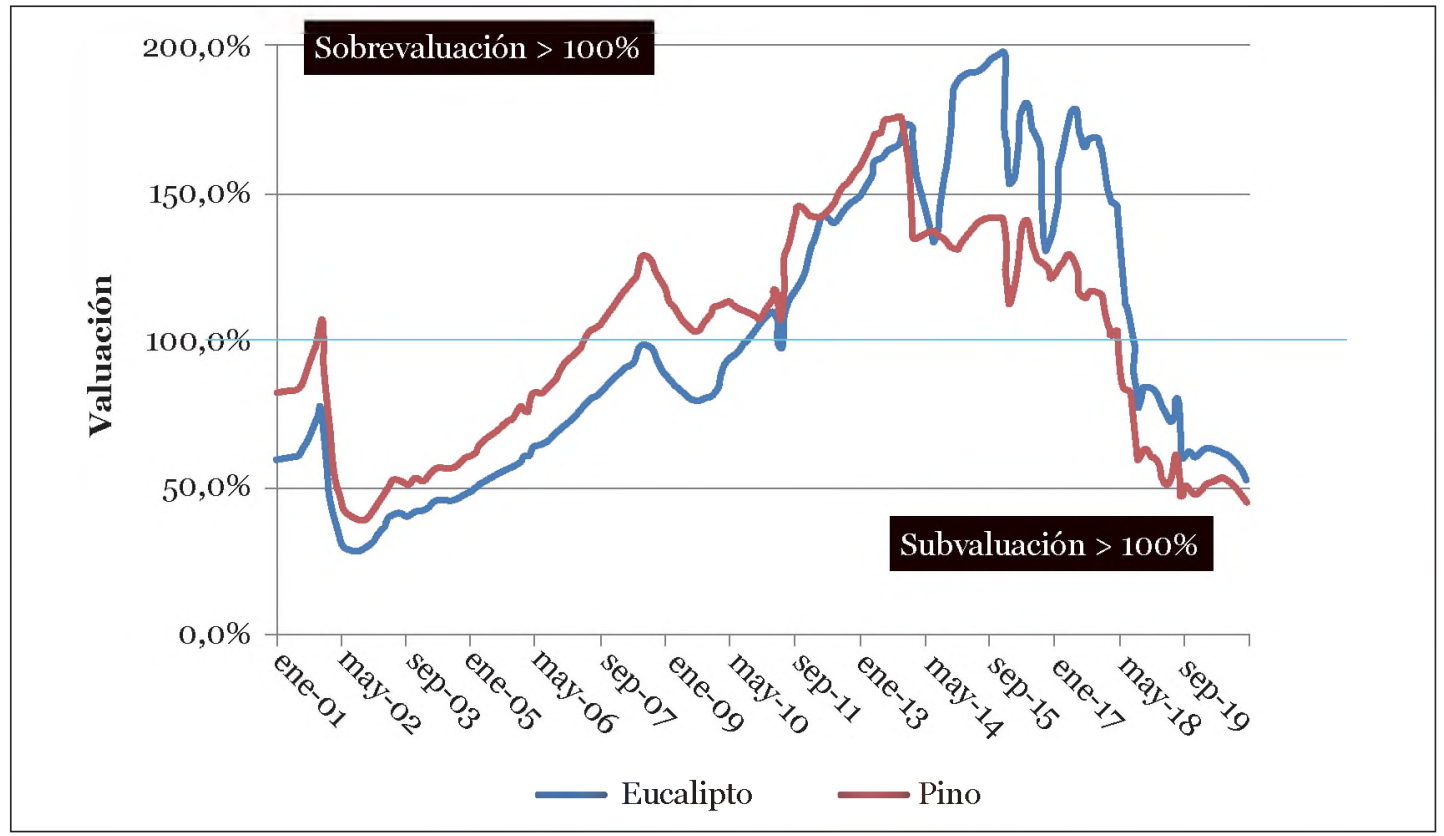

Fuente: Elaboración en base a Figuras 1 y 2.

$\mathrm{Al}$ realizar el análisis de tendencia que muestra la serie de largo plazo (Figura 3 a y 3b), se observa un movimiento creciente con pendiente 0,081 en eucalipto y 0,029 en pino, que se mantiene en toda la serie.

Ciclo: evolución de los precios que puede tener duración variable. 
Figura 3a. Tendencia y Ciclo para Eucalipto

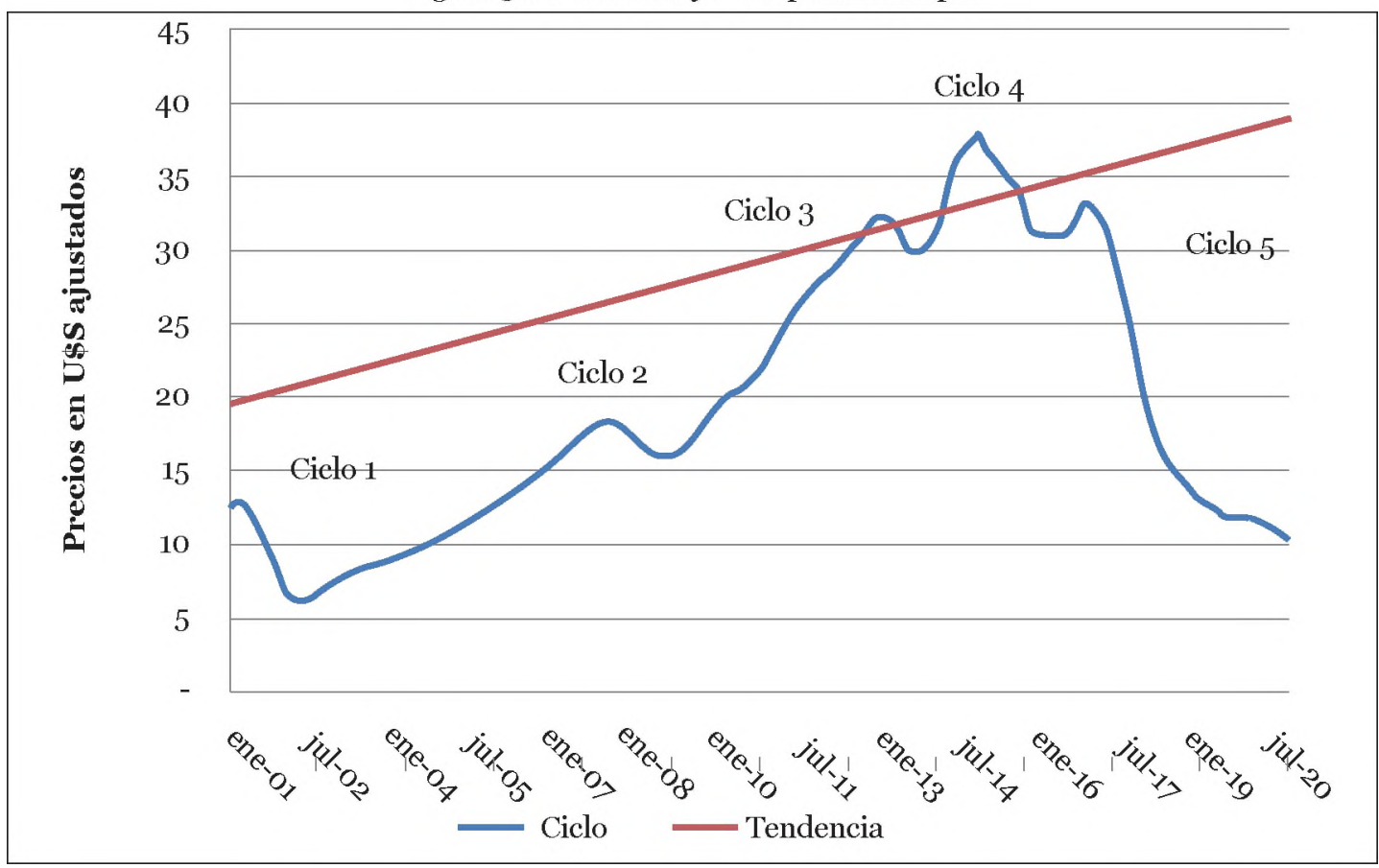

Fuente: elaboración propia.

Figura 3 b. Tendencia y Ciclo para Pino

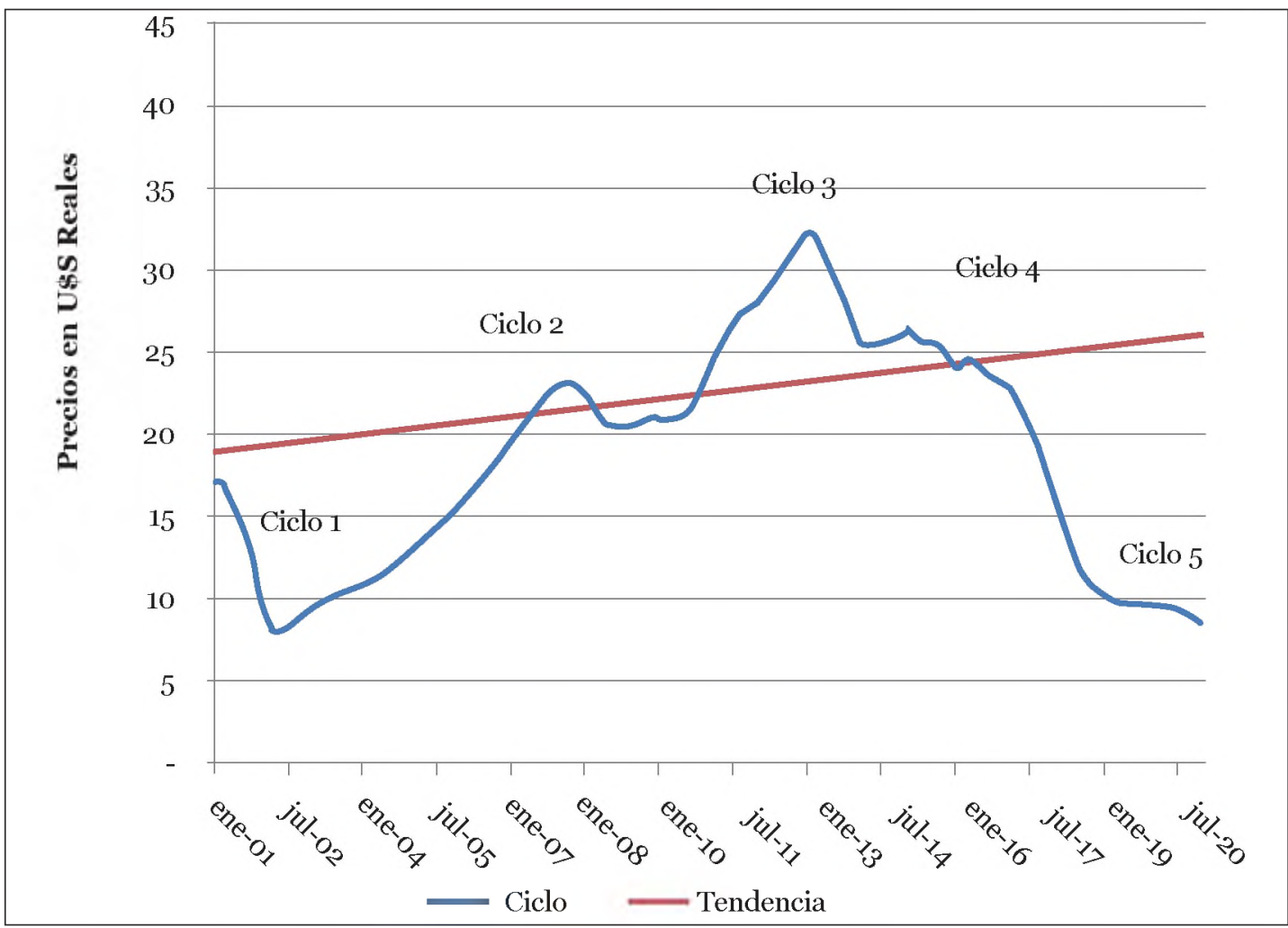

Fuente: elaboración propia. 
Por último, está el componente volatilidad. Es la división del precio, con respecto a la tendencia y el ciclo, es el residuo que queda del análisis estadístico. Figura 4a y 4b.

Figura 4a. Volatilidad de Eucalipto

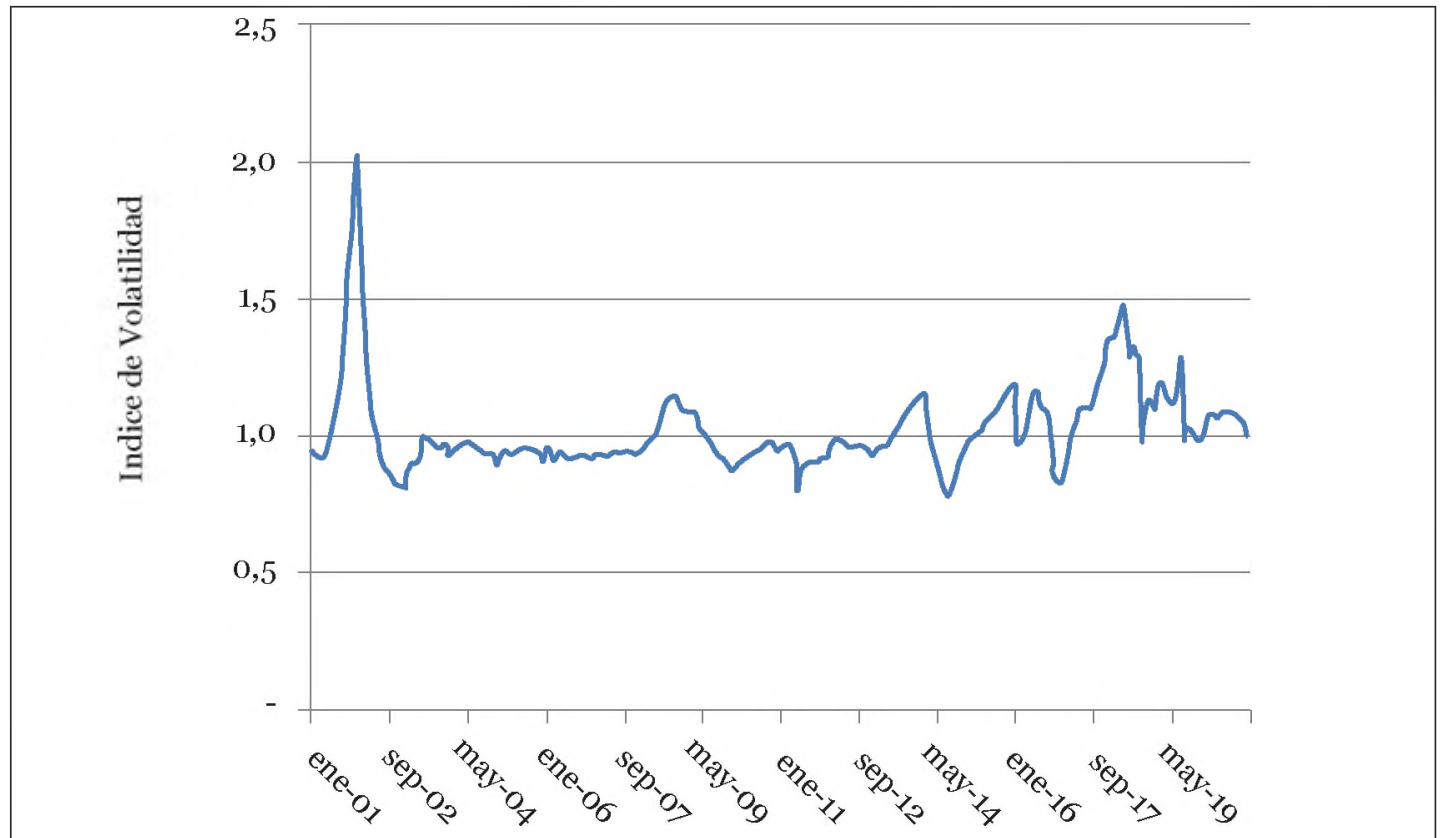

Fuente: en base a Figura 3 a.

Figura 4b. Volatilidad de Pino

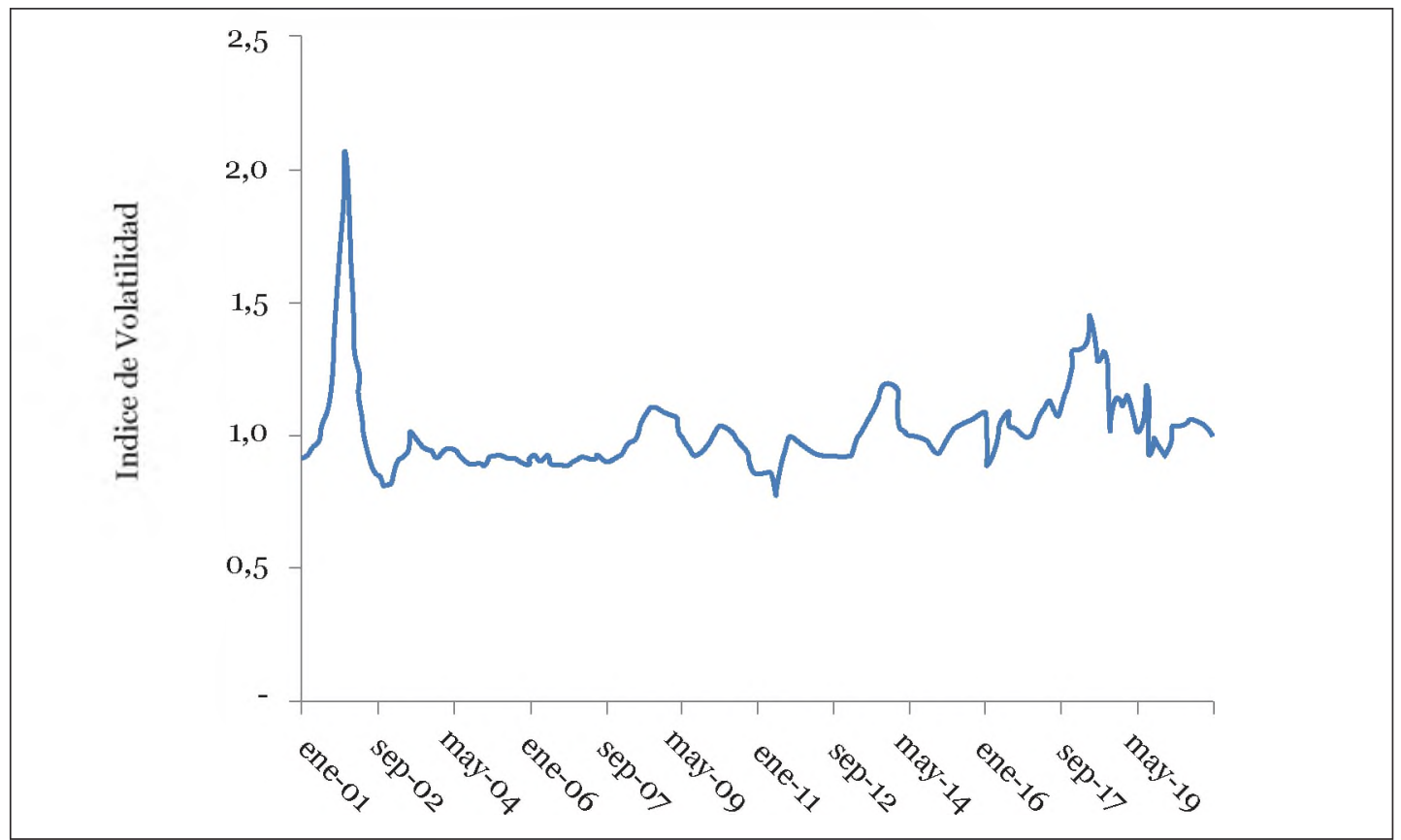

Fuente: en base a Figura 3 b. 


\section{DISCUSIÓN}

Los precios a largo plazo por tonelada en pie para eucalipto y pino son U\$S 19,55 y U\$S 18,97, respectivamente. Los cuales muestran períodos de sobrevaluación (2001-2010 y 20192020); y subvaluación (2011 y 2018), para estos bienes intermedios.

Por otro lado, el componente ciclo, está constituido por fluctuaciones ondulatorias, cuyas duraciones pueden rondar entre 3 y 5 años en cinco ciclos.

Los ciclos no poseen periodicidades iguales. Sin embargo, pueden ser difíciles de identificar, por lo que debe ser analizada junto a la tendencia, generalmente es común considerar el movimiento ciclo- tendencia de una serie.

Por último, el componente volatilidad, este consiste en variaciones sin patrón regular o identificable, ya que corresponde a la parte de la serie de tiempo que se comporta aleatoriamente. La tendencia no muestra un patrón regular de comportamiento, ya que los precios suben, se mantienen o bajan imprevistamente.

\section{CONCLUSIONES}

Argentina cuenta con 1.3 millones de hectáreas de bosque implantadas, compuestos por pinos (Pinus sp.), eucaliptos (Eucalyptus sp.), sauces (Salix sp.) y álamos (Populus sp.), de los cuales el 78,2\% se concentra en las provincias de Misiones, Corrientes y Entre Ríos. En cuanto al pino, de las 860 mil has un $82.2 \%$ se encuentra en esta región, y en eucalipto, de las 314 mil has un $87,8 \%$.

Presentamos un estudio estadístico de la serie de tiempo de los precios de dos productos forestales de amplia importancia en nuestra economía regional, el precio por tonelada en pie de eucalipto y pino en Concordia para el período 2001-2020.

Los precios son representativos del Noreste entrerriano y son la primera etapa en el análisis de la cadena de valor.

Con una base de datos relevados por el INTA EEA Concordia, se analizó el comportamiento estacional de precios de monte en pie de eucalipto y pino con corteza en el período 2001-2020. La primera consecuencia es que con un plazo de veinte años o largo plazo, podemos ver como madura la inversión realizada por nuestros productores.

Los precios históricos se convirtieron a su cotización del dólar estadounidense, ajustados por el índice de precios al consumidor de EEUU, obteniendo una serie de tiempo de valores reales.

En la revisión bibliográfica el primer antecedente fue Brescia y Rivera (2000) para frutas y hortalizas para un período de 15 años. 
El análisis de tendencia que muestra la serie de largo plazo, se observa un movimiento creciente con pendiente 0,081 en eucalipto y 0,029 en pino, que se mantiene en toda la serie. La tendencia es positiva, lo que permite esperar precios crecientes en el largo plazo.

El período estudiado se compone de 5 ciclos con una duración entre 3 y 5 años. Los ciclos no poseen periodicidades iguales. El componente estacional muestra las fluctuaciones de un precio forestal dentro de un año calendario.

El componente estacionalidad, formado por variaciones sin patrón regular o identificable. La tendencia no muestra un patrón regular de comportamiento, ya que los precios suben, se mantienen o bajan imprevistamente.

Por último, la volatilidad. Es la división del precio, con respecto a la tendencia, el ciclo y la estacionalidad

Este análisis es parte del estudio de mercado del Proyecto de Inversión Forestal.

La metodología empleada puede ser utilizada para otros productos regionales, como frutales y hortalizas.

\section{REFERENCIAS BIBLIOGRÁFICAS}

Brescia, V. y Rivera. I. (2000). El componente estacional en la comercialización de frutas y hortalizas: 1985-1999. Documento de Trabajo No 12. Instituto de Economía y Sociología (IES) del INTA. CABA. 28 p. https://inta.gob.ar/sites/default/files/script-tmp-dt_12.pdf(o9/11/2020).

Gujarati, D. y Porter, D. (2010).Econometría. $5^{\text {a }}$ Edición. McGraw Hill. México. 946 p. https:// www.academia.edu/33064534/Gujarati_Econometr\%C3\%ADa_5ta_Edici\%C3\%B3n_pdf (09/11/2020).

Hanke, J.y Wichern, D. (2010). Pronósticos en los negocios. $9^{\text {a }}$ Edición. Prentice Hall. México. $576 \mathrm{p}$.

Hoffmann, R. (2006). Estadística para economistas. CENGAGE. Sao Paulo. 427 p. https:// es.scribd.com/document/402663779/291025232-Estatistica-para-Economistas-Hoff-pdf-pdf (09/11/2020).

Ministerio de Agricultura Ganadería y Pesca de la Nación Argentina. (2015). Dirección de Producción Forestal. Publicación: Argentina: Plantaciones Forestales y Gestión Sostenible. Disponible en: http://forestoindustria.magyp.gob.ar/archivos/gestion-forestal-sostenible/ publi_ambiental.pdf (09/11/2020). 
Ministerio de Hacienda. (2019). Informe de Cadena de Valor Forestal. Año $4 \mathrm{~N}^{\circ} 1438$ p. https://www.argentina.gob.ar/sites/default/files/sspmicro_cadenas_de_valor_forestal_ papel_muebles.pdf (09/11/2020).

Molina, N. y Ríos de González, L. (2019). Tendencia, Ciclo y Estacionalidad del limón en el Mercado de Concentración de Corrientes. Período 2004-2018. Serie Técnica 69. INTA EEA Bella Vista. 30 p.

Morettin, P. y Toloi, C. (2006). Análise de Séries Temporais. Editorial Blucher. San Pablo. 540 p. https://es.scribd.com/document/346974880/Analise-de-Series-temporais-Morettin-pdf (09/11/2020).

Peirano, C.; Bussio, D.; Beccar Varela, A. (2019). Elaboración del plan estratégico forestal y forestoindustrial Argentina 2030. Secretaría de Ambiente y Desarrollo Sustentable, Ministerio de Agricultura, Ganadería y Pesca, Ministerio de Educación, Cultura, Ciencia y Tecnología Presidencia de la Nación Argentina, disponible en: https://www.agroindustria.gob.ar/sitio/ areas/sycf/publicacion-forestales-11-diciembre-2019.pdf (09/11/2020).

Vera, L. (2020). Estimaciones de costos, precios y resultados en la actividad forestal en el Noreste de Entre Ríos de los 200O-2020. XXXIV Jornadas Forestales de Entre Ríos. INTA EEA Concordia. https://www.jornadasforestales.com.ar/book/revista/jornadas-forestales2020.pdf (09/11/2020).

Vera, L.; Mastandrea, C., Peña, C., Vianna, M. y Palenzona, M. (2020). Planilla de precios forestales de la región NE de Entre Ríos. INTA EEA Concordia. https://inta.gob.ar/ documentos/planilla-de-precios-forestales-de-la-region-ne-de-entre-rios (09/11/2020).

Wei, W. (2006). Time Series Analysis. Univariate and Multivariate Methods. Second Edition. Pearson. USA. 634 p. http://civil.colorado.edu/ balajir/CVEN6833/lectures/wwts-book.pdf (09/11/2020). 


\section{CURRICULUM VITAE}

\section{Néstor Albino Molina}

Contador Público Nacional, Facultad de Ciencias Económicas, Universidad Nacional del Nordeste, Argentina. Licenciado en Economía, Facultad de Ciencias Económicas, Universidad Nacional del Nordeste, Argentina. Especialista en Administración y Planificación del Desarrollo Regional: CIDER de la Universidad de los Andes, Colombia. Magister en Marketing Internacional: Posgrado de la Facultad de Ciencias Económicas, Universidad Nacional del Litoral, Argentina. Doctor en Economía, Universidad Nacional de Rosario, Argentina.

molina.nestor@inta.gob.ar

\section{Luis María Mestres}

Ingeniero Forestal, Universidad Nacional de La Plata, Argentina.

mestres.luis@inta.gob.ar 